\title{
Association between concomitant psychiatric drug use, and patients' beliefs about and persistence with chronic cardiovascular medication
}

\author{
L. Hromadkova, ${ }^{1,2}$ E. R. Heerdink, ${ }^{2}$ D. Philbert, ${ }^{2}$ M. L. Bouvy ${ }^{2}$
}

'Department of Clinical Pharmacy, Hospital Na Homolce, Prague, Czech Republic

${ }^{2}$ Division Pharmacoepidemiology and Clinical Pharmacology, Faculty of Science, Utrecht University, Utrecht, The Netherlands

Correspondence to: Lucie Hromadkova, Department of Clinical Pharmacy, Hospital Na Homolce, Roentgenova 37/

2, 15000 Prague, Czech Republic

Tel.: +420723904581 Email: lucie.hromadkova@ gmail.com

Disclosures

None of the authors do have any conflict of interests.

\section{SUMMARY}

Objective: Psychiatric disorders are associated with an increased risk of cardiovascular diseases and may result in additional risk of non-adherence. No data on the influence of concomitant psychiatric drug use on patients' beliefs and persistence related to cardiovascular medication are available. The objective of this study was to assess to what extent the use of concomitant psychiatric drugs is associated with patients' beliefs about and persistence with chronic cardiovascular medication. Methods: An observational study in patients using cardiovascular medication was conducted. A mailed questionnaire containing socio-demographical questions and a measure of beliefs about medication (Beliefs about Medicines Questionnaire - specific) was sent to patients selected from fifteen participating pharmacies. Persistence was evaluated based on pharmacy records. Results: of the 1547 included patients, 551 responded to key questions in the questionnaire and were included for beliefs about medication analysis. In concomitant users of psychiatric drugs significantly higher necessity (17.0 vs. 16.0) and higher concerns (14.3 vs. 13.3), as well as higher proportion of ambivalent (34.5\% vs. $25.6 \%)$ and lower proportion of indifferent patients (24.1\% vs. $33.0 \%)$ were found compared with non-users $(p<0.05) .65 .2 \%(n=1009)$ of patients were persistent on all their cardiovascular drugs. There was no significant association between concomitant use of psychiatric drugs and non-persistence $(O R=1.2$; $95 \%$ Cl 0.9-1.5). Conclusion: Concomitant use of psychiatric drugs was found to be associated with increased beliefs about the necessity of and concerns about cardiovascular medication. Clinicians caring for cardiovascular patients should give additional attention to identifying patients' beliefs about medication among those concomitantly using psychiatric drugs.

\section{What's known}

There is an increased risk of cardiovascular diseases in psychiatric disorders. It is also known that psychiatric disorders may result in additional risk of non-adherence to psychiatric drugs. Nevertheless, no data on the influence of concomitant psychiatric drug use on patients' beliefs and persistence related to cardiovascular medication are available.

\section{What's new}

In concomitant users of psychiatric drugs significantly higher necessity of and higher concerns about cardiovascular medication were found compared with non-users. No significant association between concomitant use of psychiatric drugs and nonpersistence on cardiovascular medication was found.

\section{Introduction}

Cardiovascular diseases are the leading cause of mortality in Europe (almost 4.1 million deaths per year, or $46 \%$ of all deaths in Europe) (1). Therefore, in patients with a higher cardiovascular risk chronic preventive treatment is recommended (2). One of the key requirements for successful treatment is patient adherence and persistence with medication. However, in patients with chronic cardiovascular treatment poor adherence to prescribed medications is common and non-adherence is associated with increased mortality (3). In a previous study patients' beliefs about their chronic medication were found to be a strong predictor of patient adherence, and patients with stronger beliefs about their medication reached better adherence (4).

It is known that psychiatric disorders are associated with an increased risk of coronary heart disease. The prevalence of cardiovascular diseases in people with schizophrenia and bipolar disorder is approximately two to threefold increased $(5,6)$. It was presented that depression is one of the demonstrable risk factors for the development of cardiovascular diseases (7-9). Association between chronic anxiety and increased risk of coronary heart disease has also been shown, and it has been suggested that in patients with schizophrenia other risk factors for coronary heart disease (smoking, obesity, diabetes mellitus, hypertension, high blood cholesterol) are 
more common than in the general population $(10,11)$. Given these facts, the concomitant use of psychiatric and cardiovascular drugs may be quite frequent in practice. Nevertheless, most studies focused on cardiovascular drug use in psychiatric patients have found a slightly reduced use of $\mathrm{CV}$ medication (12-15), while others did not find such a reduction $(16,17)$. In a large epidemiological study in 2463 patients with schizophrenia, $10.9 \%$ of patients were treated for arterial hypertension and $7.1 \%$ for a lipid disorder. In addition, 70\% of untreated patients presented biochemical evidence of dyslipidaemia, 39\% had hypertension and a metabolic syndrome was identified in $34 \%$ (13). In another study a 0.37 -fold use of antihypertensive and 0.47 -fold use of lipid-lowering drugs was found in psychiatric patients compared with the general population (15). One of the possible explanations for the undertreatment might be low drug adherence of psychiatric patients.

Psychiatric comorbidities may result in an additional risk of non-adherence. Drug adherence is difficult to achieve among patients with schizophrenia or anxiety disorders $(11,18)$. In addition, depression has been shown to be associated with poor adherence in patients with coronary heart disease $(19,20)$. Nevertheless, it is unclear whether patients who are treated for their depression are still at higher risk of non-adherence. In patients with acute coronary syndrome a subsequent improvement in adherence to acetylsalicylic acid after reduction in depressive symptoms was shown (21). In general, patients with a psychiatric diagnosis and users of a higher number of psychiatric drugs are usually at higher risk of non-adherence. It is unclear whether the dominant role is played by the psychiatric illness itself or by the effects of psychiatric medication on cognition (and thus beliefs). Therefore, in our research we defined psychiatric patients as users of psychiatric drugs to capture both possibilities.

It has never been tested whether concomitant use of psychiatric drugs influences patients' beliefs about cardiovascular medication and whether this potential influence has any impact on patient adherence to cardiovascular medication. In general, persistence is considered as a part of adherence, usually as the most extreme form because non-persistence means that the medication is no longer taken at all. Therefore, we aimed to work with that form of adherence in our research and the aim of our study was to assess to what extent the use of concomitant psychiatric drugs is associated with patients' beliefs about and persistence with chronic cardiovascular medication.

\section{Methods}

\section{Setting}

In patients using cardiovascular medication a retrospective observational study combined with a crosssectional survey was conducted. Patients $\geq 18$ years of age with at least one prescription for cardiovascular medication within the 3 months before the selection were identified from community pharmacy dispensing records. Dispensing records were obtained from 15 pharmacies, which were selected from 58 Dutch pharmacies willing to participate in this study. In the selection, we tried to achieve diversity in terms of the degree of urbanisation, percentage of second generation immigrants and percentage of patients aged over 65 years. Data were obtained from Statistics Netherlands (www.cbs.nl). More details are available in our previous study (22). The cardiovascular medications of our interest were antihypertensive drugs (ATC C02, C03, C07, C08, C09) and statins $(\mathrm{C} 10)$ because they represent the key therapy for modifying of overall cardiovascular risk and are recommended for patients with a 10-year mortality risk for cardiovascular disease of over 10\% (2). The selected patients were sent a mailed questionnaire and after 3 weeks, a reminder card was sent. For all selected patients, the pharmacy dispensing records spanning the last 1 year were extracted and anonymised with a study ID. The study was approved by the Institutional Review Board of the division of Pharmacoepidemiology and Clinical Pharmacology of Utrecht University.

\section{Beliefs about medicines}

Patients received a mailed questionnaire that consisted of general questions focused on patients' socio-demographical and health status and of the Beliefs about Medicines Questionnaire (BMQ) (23). General questions regarded gender, age, height, weight, education and smoking status of patients. The BMQ was used to measure patients' beliefs about cardiovascular medicines (BMQ-specific) and for our purposes standard forward/backward translation and cross-cultural adaptation was performed according to the recent guidelines (24). The BMQ evaluates two components of patient's beliefs: patients' necessity of prescribed medications (necessity scale) and patients' concerns about the prescribed medications (concerns scale). The scale scores, ranging from 5 to 25 , were calculated from individual items of the BMQ scored using a 5-point Likert scale $(1=$ strongly disagree, $2=$ disagree, $3=$ uncertain, $4=$ agree, $5=$ strongly agree). Higher scores on the scales indicate stronger beliefs in the concepts represented by the scale. The necessity and 
concerns scales were split at the median to generate four attitudinal groups: accepting (high necessity, low concerns), ambivalent (high necessity, high concerns), indifferent (low necessity, low concerns), sceptical (low necessity, high concerns).

\section{Persistence}

Persistence with cardiovascular drugs was evaluated based on the pharmacy records. It was defined as a dichotomous variable (persistent vs. non-persistent) based on the presence of an expected gap in drug intake. The gap was represented by a break of 30 or more days in drug use. Although, there is no clear consensus regarding the ideal gap length for determination of treatment discontinuation $(25,26)$, the 30 day gap is commonly used in the literature $(27,28)$. Moreover, we considered this gap length as appropriate for our purposes and as a good compromise between the ability to detect non-persistence and maximum tolerated time without cardiovascular medicine. Observation period for evaluation was 1 year before the selection of patients and before completion of the questionnaire. First, persistence on individual cardiovascular drugs was assessed. Patients were considered as persistent on the individual drug, when there was no gap in drug intake during the observation period. Based on the persistence on individual drugs an evaluation of overall persistence was performed. Patients were considered to be overall persistent in the case of persistence on all used cardiovascular drugs. The group of non-persistent patients, i.e. of patients with a gap in intake of at least one cardiovascular drug, was further evaluated in total but also divided into two categories of patients non-persistent on one drug and patients non-persistent on $\geq 2$ drugs. The number of drugs was determined using unique ATC codes.

\section{Psychiatric drugs}

The use of psychiatric drugs was identified from the pharmacy records. Observation period was 1 year before the selection of patients, i.e. before questionnaire completion. Antidepressants, benzodiazepines and antipsychotics were psychiatric drugs of our interest. Patients were considered as users of psychiatric drugs, when there was at least one prescription of these drugs during the observation period. Patients were also stratified into three categories based on the number of different psychiatric drugs used (no psychiatric drug, 1 and $\geq 2$ psychiatric drugs).

\section{Statistical methods}

The group of respondents was investigated to determine the relationship between the use of psychiatric drugs and patients' beliefs about cardiovascular medicines. Socio-demographical and health characteristics of respondents and their beliefs about cardiovascular medicines were compared among users and non-users of psychiatric drugs. Beliefs about cardiovascular medicines were assessed as separate scales (necessity and concerns scale) and also based on the four attitudinal belief groups. To identify the relationship between patients' beliefs and the use of psychiatric drugs univariate and multivariate logistic regression models were examined. Every belief group was compared with the three other remaining groups merged together. Even though patients' characteristics did not differ significantly between users and non-users in univariate logistic regression, they were considered to be potential confounders and stepwise multivariate logistic regression models were used. Every BMQ outcome, i.e. both scales and all belief groups, were entered into this model separately.

All patients (respondents and non-respondents) were investigated to determine the association between the use of psychiatric drugs and persistence on cardiovascular drugs. Univariate logistic regression was performed and all characteristics were further included in the multivariate logistic regression model. The use of psychiatric drugs and patient categories based on the number of psychiatric drugs were included separately. First, persistent and non-persistent patients were compared and in the next step the analysis included patients divided into three categories of persistence, i.e. persistent patients, patients non-persistent on one drug and patients non-persistent on $\geq 2$ drugs.

Data were analysed using sPSs for Windows, version 19.

\section{Results}

\section{Population characteristics}

Of the total number of patients $(n=1547)$ included in our study, 551 patients $(35.6 \%)$ responded to the key questions of the BMQ. Data regarding sociodemographical, health and medication characteristics of all patients and respondents that filled in the key questions of the BMQ are summarised in Table 1.

Comparing respondents $(n=551)$ and nonrespondents $(n=996)$ (data not shown in tables), no significant difference in mean age was found (62.7 vs. 61.5), but the proportion of patients in individual age groups differed significantly. Respondents were underrepresented in the 18-50-year $(\mathrm{p}<0.001)$ and $>70$-year $(\mathrm{p}<0.05)$ age group and overrepresented in the 61-70-year age group $(\mathrm{p}<0.001)$. Furthermore, respondents less frequently 
Table 1 Socio-demographical and medication characteristics of patients: all patients $(n=1547)$ and respondents to BMQ key questions $(n=551)$

\begin{tabular}{|c|c|c|}
\hline Patient characteristics & $\begin{array}{l}\text { All patients } \\
(n=1547) \\
\%(n)\end{array}$ & $\begin{array}{l}\text { Respondents } \\
(n=551) \\
\%(n)\end{array}$ \\
\hline \multicolumn{3}{|l|}{ Gender } \\
\hline Female & $52.2(807)$ & $47.2(260)$ \\
\hline \multicolumn{3}{|l|}{ Age } \\
\hline $18-50$ & $20.6(318)$ & $14.3(79)$ \\
\hline $51-60$ & $23.4(362)$ & $26.1(144)$ \\
\hline $61-70$ & $25.3(391)$ & $32.1(177)$ \\
\hline$>70$ & $30.8(476)$ & $27.4(151)$ \\
\hline \multicolumn{3}{|l|}{ Cardiovascular drugs use } \\
\hline Beta-blockers & $56.9(880)$ & 57.5 (317) \\
\hline Calcium channel blockers & $27.7(429)$ & $27.9(154)$ \\
\hline ACE inhibitors & $34.7(537)$ & 36.1 (199) \\
\hline $\mathrm{AT}_{2}$ blockers & $24.4(378)$ & $25.2(139)$ \\
\hline Diuretics & $35.0(541)$ & $37.0(204)$ \\
\hline Other antihypertensives & $1.1(17)$ & $1.5(8)$ \\
\hline Statins & $50.4(779)$ & $54.3(299)$ \\
\hline \multicolumn{3}{|l|}{ Psychiatric drugs use } \\
\hline Antidepressants & $13.4(207)$ & $10.3(57)$ \\
\hline Benzodiazepines & $23.6(365)$ & $20.7(114)$ \\
\hline Antipsychotics & $3.6(56)$ & $1.5(8)$ \\
\hline $\begin{array}{l}\text { Number of psychiatric } \\
\text { drugs users }\end{array}$ & $30.4(470)$ & $26.3(145)$ \\
\hline \multicolumn{3}{|l|}{ Education level } \\
\hline Non/primary school & - & $38.4(208)$ \\
\hline Secondary school & - & $40.6(220)$ \\
\hline College/University & - & $21.0(114)$ \\
\hline \multicolumn{3}{|l|}{ BMI } \\
\hline Underweight (< 18.5) & - & $0.4(2)$ \\
\hline Normal range (18.5-25) & - & $32.7(176)$ \\
\hline Overweight (25-30) & - & $43.9(236)$ \\
\hline Obese $(>30)$ & - & $23.0(124)$ \\
\hline \multicolumn{3}{|l|}{ Smoking behaviour } \\
\hline Current smoker & - & $15.8(87)$ \\
\hline Ex-smoker & - & $50.1(275)$ \\
\hline Lifelong non-smoker & - & 34.1 (187) \\
\hline
\end{tabular}

used psychiatric drugs as concomitant medication $(26.3 \%$ vs. $32.6 \%$; $p<0.05)$, the difference was found in the use of all three groups of psychiatric drugs (antidepressants, benzodiazepines and antipsychotics). There were no significant differences between respondents and non-respondents with regard to the use of cardiovascular medication, except for the more frequent use of statins by respondents $(54.3 \%$ vs. $48.2 \%$; p $<0.05)$. The proportion of persistent patients was significantly higher in the group of respondents $(68.8 \%$ vs. $63.3 \%$; $\mathrm{p}<0.05)$.

\section{Beliefs about cardiovascular medication}

The mean scores $( \pm S D)$ of the necessity and concerns BMQ scales of the 551 respondents were 16.3 $( \pm 4.3)$ and $13.6( \pm 4.1)$, respectively. Users of psychiatric drugs had significantly higher necessity (17.0 vs. 16.0) and also significantly higher concerns (14.3 vs. 13.3) scores compared with non-users of psychiatric drugs $(\mathrm{p}<0.05)$. Based on the evaluation of belief groups, a significantly higher proportion of ambivalent (34.5\% vs. $25.6 \%$ ) and lower proportion of indifferent $(24.1 \%$ vs. $33.0 \%)$ patients was found in users of psychiatric drugs compared with nonusers $(p<0.05)$. Adjustment for other patient characteristics had no influence on the results of BMQ outcomes. Details about the relationship between psychiatric drug use and BMQ outcomes are shown in Table 2.

\section{Persistence}

Nearly two-thirds of patients $(65.2 \% ; n=1009)$ were persistent on all of their cardiovascular drugs. In the group of non-persistent patients, a higher proportion of users of psychiatric drugs was found compared with persistent patients (32.5\% vs. 29.2\%; non-significant). A similar trend was also seen in separate evaluation of users of antidepressants or benzodiazepines, but in users of antipsychotics an opposite trend was found (none of these results were significant, data not shown in tables). No significant differences in gender or age were found between persistent and non-persistent patients, but non-persistent patients used a significantly larger number of cardiovascular drugs (1.96 vs. 2.94; $\mathrm{p}<0.001)$.

A multivariate logistic regression model containing all variables showed a higher but non-significant risk to be non-persistent (adjusted OR 1.2; 95\% CI 0.9 $1.5)$ in users of psychiatric drugs. A significant association between the number of cardiovascular drugs used by patients and their persistence was found with two times greater risk for each additional cardiovascular drug used (adjusted OR 2.0; 95\% CI 1.8-2.1; $\mathrm{p}<0.001$ ). There was an increased (but not significant) risk of non-persistence with increasing number of psychiatric drugs used and this risk was higher (but still not significant) in users of $\geq 2$ drugs (compared with non-users). Details are shown in Table 3.

Furthermore, more detailed analysis among three categories of persistence (persistent: $n=1009$, non-persistent on one drug: $n=369$, non-persistent on $\geq 2$ drugs: $n=169$ ) was performed (data not shown in tables). A trend of increasing proportions of psychiatric drugs users among these three categories of persistence, from persistent to the most 
Table 2 Relationship between patients' beliefs and psychiatric drug use $(n=551)$

\begin{tabular}{|c|c|c|c|}
\hline & \multicolumn{2}{|c|}{$\begin{array}{l}\text { Psychiatric drug use } \\
\text { (BZDs, antidepressants, antipsychotics) \% ( } n \text { ) }\end{array}$} & \multirow[b]{2}{*}{$\begin{array}{l}\text { OR }(95 \% \mathrm{Cl}) \\
\text { crude }\end{array}$} \\
\hline & $\begin{array}{l}\text { Non-users } \\
73.7 \% \text { (406) }\end{array}$ & $\begin{array}{l}\text { Users } \\
26.3 \% \text { (145) }\end{array}$ & \\
\hline Female & $45.3(184)$ & $52.4(76)$ & $1.33(0.91-1.92)$ \\
\hline Age (mean \pm SD) & $62.23 \pm 12.33$ & $64.07 \pm 11.18$ & $1.01(0.99-1.03)$ \\
\hline \multicolumn{4}{|l|}{ Education } \\
\hline Non/primary school & $38.8(155)$ & $37.1(53)$ & Reference \\
\hline Secondary school & $38.8(155)$ & $45.5(65)$ & $1.23(0.80-1.88)$ \\
\hline College/University & $22.3(89)$ & $17.5(25)$ & $0.82(0.48-1.41)$ \\
\hline \multicolumn{4}{|l|}{ Smoking } \\
\hline Smokers & $14.6(59)$ & $19.4(28)$ & $1.27(0.73-2.20)$ \\
\hline Ex-smokers & $51.9(210)$ & $45.1(65)$ & $0.83(0.54-1.26)$ \\
\hline Lifelong non-smokers & $33.6(136)$ & $35.4(51)$ & Reference \\
\hline \multicolumn{4}{|l|}{ BMI } \\
\hline Normal range (18.5-25) & $33.9(135)$ & $29.3(41)$ & Reference \\
\hline Overweight (25-30) & $44.5(177)$ & $42.1(59)$ & $0.91(0.58-1.43)$ \\
\hline Obese $(>30)$ & $21.4(85)$ & $27.9(39)$ & $1.51(0.90-2.53)$ \\
\hline \multicolumn{4}{|l|}{ BMQ - beliefs groups } \\
\hline Accepting & $20.4(83)$ & $21.4(31)$ & $1.06(0.66-1.68)$ \\
\hline Ambivalent & $25.6(104)$ & $34.5(50)$ & $1.53(1.02-2.30)$ \\
\hline Sceptical & $20.9(85)$ & $20.0(29)$ & $0.94(0.59-1.51)$ \\
\hline Indifferent & $33.0(134)$ & $24.1(35)$ & $0.65(0.42-0.99)$ \\
\hline \multicolumn{4}{|c|}{ BMQ - scales (mean \pm SD) } \\
\hline N-scale & $16.02 \pm 4.2$ & $17.02 \pm 4.3$ & $1.06(1.01-1.11)$ \\
\hline C-scale & $13.32 \pm 4.0$ & $14.26 \pm 4.2$ & $1.06(1.01-1.11)$ \\
\hline \multicolumn{4}{|l|}{ Medication (mean $\pm S D$ ) } \\
\hline No of CV drugs & $2.51 \pm 1.34$ & $2.35 \pm 1.24$ & $0.91(0.78-1.05)$ \\
\hline
\end{tabular}

non-persistent patients $(29.2 \% ; n=295$ vs. $31.2 \%$; $n=115$ vs. $35.5 \% ; n=60$, respectively), was found. After adjusting for other variables (age, gender and number of cardiovascular drugs used) the difference between two marginal groups (persistent vs. non-persistent on $\geq 2$ drugs) remained non-significant, with a trend towards higher risk (OR 1.5; 95\% CI 1.02.3 ) to be non-persistent on $\geq 2$ cardiovascular drugs in users of psychiatric drugs.

\section{Discussion}

In this study, the influence of the usage of psychiatric drugs (antidepressants, benzodiazepines and antipsychotics) on patients' beliefs about chronic cardiovascular treatment was investigated and the impact on persistence was evaluated. We found that the use of psychiatric drugs is significantly associated with patients' beliefs which may have an influence on patient persistence, although this was not significant in this study.
In our results users of psychiatric drugs differed in both components of beliefs about cardiovascular medication from non-users. When comparing the individual BMQ scales scores between users of psychiatric drugs and non-users, necessities and concerns scores of users were significantly increased. The same result was found when comparing proportional representations of patients in belief groups. In the group of psychiatric drug users, a significantly higher proportion of ambivalent patients (high necessity, high concerns) and lower proportion of indifferent patients (low necessity, low concerns) was found. We were unable to find any study dealing with influence of psychiatric drug use on beliefs about chronic cardiovascular medication. There are some studies performed in psychiatric or cardiac patients investigating beliefs about medication for these disorders separately. In the original UK sample used in the BMQ validation study, psychiatric (and also asthmatic) patients had significantly higher concerns about their psychiatric medication than other illness 
Table 3 Association between persistence and psychiatric drug use $(n=1547)$

\begin{tabular}{|c|c|c|c|c|}
\hline & $\begin{array}{l}\text { Persistent } \\
65.2 \%(n=1009) \\
\%(n)\end{array}$ & $\begin{array}{l}\text { Non-persistent } \\
34.8 \%(n=538) \\
\%(n)\end{array}$ & OR $(95 \% \mathrm{Cl})$ crude & OR $(95 \% \mathrm{Cl})$ adjusted \\
\hline Female & $52.1(526)$ & $52.2(281)$ & $1.00(0.81-1.23)$ & $0.88(0.70-1.11) *$ \\
\hline Age (mean \pm SD) & $62.11 \pm 14.44$ & $63.35 \pm 14.61$ & $1.01(1.00-1.01)$ & $0.99(0.98-1.00) *$ \\
\hline Psychiatric drug use & $29.2(295)$ & $32.5(175)$ & $1.17(0.93-1.46)$ & $1.21(0.94-1.54) \dagger$ \\
\hline \multicolumn{5}{|l|}{ No of psychiatric drugs } \\
\hline 0 & $70.8(714)$ & $67.3(362)$ & Reference & Reference \\
\hline 1 & $18.3(185)$ & $19.5(105)$ & $1.12(0.85-1.47)$ & $1.10(0.82-1.48) *$ \\
\hline$\geq 2$ & $10.9(110)$ & $13.2(71)$ & $1.27(0.92-1.76)$ & $1.40(0.99-2.00) *$ \\
\hline No of CV drugs (mean \pm SD) & $1.96 \pm 1.10$ & $2.94 \pm 1.31$ & $1.91(1.74-2.09)$ & $2.00(1.82-2.12) *$ \\
\hline
\end{tabular}

groups but also lower necessities (23). Unfortunately, the studied population of psychiatric patients was not described in more detail. Another study evaluating the beliefs about psychiatric drugs in psychiatric patients showed that users of these drugs have higher concerns about and higher necessities of their medication than users of antihypertensive medications (29).

Despite the similar results with the second and partly also with the first mentioned study, we expected an important difference in beliefs of psychiatric patients about psychiatric and about cardiovascular medication. Psychiatric patients may have a skewed perception of their psychiatric disease, but increased anxiety from their cardiovascular disease. Therefore, the difference between beliefs about cardiovascular and about psychiatric medication is probable, i.e. cardiovascular patients with psychiatric comorbidities can feel the necessity of use of cardiovascular drugs even though they do not feel any necessity of the use of their psychiatric drugs. In addition, in psychiatric patients a higher presence of anxiety, also related to health and present cardiovascular disease, is probable and therefore, our findings of higher necessities and also higher concerns in psychiatric users are explainable.

In addition to examining beliefs of psychiatric drug users about their cardiovascular medication, we investigated whether psychiatric drug use has any impact on overall patient persistence on cardiovascular medication. In our sample $65.2 \%$ of all patients were persistent on their cardiovascular treatment. A review evaluating 12-month persistence in cardiovascular and diabetic patients has shown an average persistence of $63.3 \%$, varying from $35.1 \%$ to $92.0 \%$
(30). In our study, the association between the use of psychiatric drugs and non-persistence did not reach a statistical significance. A higher risk of nonpersistence was found to be related to a higher number of cardiovascular drugs used by patients. The number of used drugs is a generally considered predictor of persistence (31-33), but there are also studies which do not confirm this finding $(34,35)$. Patients with psychiatric illness typically have great difficulty to follow a medication regimen and in some studies depression was found to be associated with non-compliance to antihypertensive medications $(33,35-37)$. It is necessary to take into consideration that the group of psychiatric drug users varies within itself. A higher risk of non-persistence in psychiatric patients was shown previously and patients with a psychiatric diagnosis and users of a higher number of psychiatric drugs are expected to be at higher risk of non-persistence (11-20). On the other hand, it can be assumed that psychiatric drug use might be related to better control of mental illness, which in turn could lead to increased compliance with cardiovascular medication. Even though in our study the slightly higher, but non-significant risk of non-persistence was found in users of psychiatric drugs in general, not all psychiatric drugs are used for psychiatric conditions. Therefore, we suppose that in the whole group of psychiatric drug users patients with higher and lower risk of non-persistence will occur. Also the number of psychiatric drugs did not influence persistence on cardiovascular drugs significantly in our study, only a trend was seen. Also differences between particular psychiatric drugs can be supposed, but our findings were not significant in this area, only trends were seen. In users of antipsychotics, the opposite trend might be 
explained by the small number of these users in our study but probably also by more frequent hospitalisation of these patients, associated with greater attention from clinicians. Further research is needed in this area.

Clinicians caring for cardiovascular patients should consider the question of possible patient non-persistence in their clinical practice, because non-persistence may decrease treatment outcomes and it increases mortality of patients (3). Based on our results, more attention should be given to patients with concomitant psychiatric drugs because in these patients higher beliefs about the importance of taking cardiovascular medication together with higher concerns about this medication was found. We assume that focusing on decrease in patient concerns might increase their persistence.

\section{Study limitations and strengths}

Because only about a third of patients responded to our questionnaire, some selection bias may have occurred. Persistence of respondents was significantly higher than persistence of non-respondents in our study. Similar results have been found before (38). However, a finding of a higher proportion of psychiatric drug users in non-respondents, as well as more non-persistence in this group, is in agreement with our results about the trend between non-persistence and psychiatric drug use. Differences between our respondents and non-respondents have no impact on the evaluation of association between persistence and psychiatric drug use, because all patients were included in this evaluation. However, the possible bias may affect the investigation of patients' beliefs. An association between patients' beliefs and adherence has been previously found (4). Because there are more non-persistent patients among non-respondents, we cannot eliminate the possibility that non-respondents' beliefs are different.

A possible second limitation is a bias in the selection of pharmacies. The pharmacies invited to participate are a part of the large research and education network consisting of about 1300 community pharmacies, which is nearly two-thirds of all pharmacies in the Netherlands. Therefore, we do not expect the pharmacies in the network to be different from other pharmacies with regard to their level of counselling that could influence patients' beliefs or persistence. In addition, the participating pharmacies cover different areas and levels of urbanisation in the Netherlands, and the selected patients represent a diverse sample of the population (22).
Further limitations of the study may be considered as lack of information on the nature of the psychiatric diagnoses and on the relative control of mental disorder. This might be the subject of future research.

The strength of our study is that it is the first investigation into the influence of psychiatric drug use on beliefs about medication for diseases other than the psychiatric ones. In the area of cardiovascular disorders, we have not found this issue to be previously investigated. In addition, in the literature not many studies evaluating the influence of psychiatric drug use on persistence occur. Another strength is the linking of data directly obtained from patients through the questionnaire with the pharmacy dispensing data. Because of the availability of the pharmacy dispensing data, it was possible to calculate patient persistence and to compare some demographical and medication characteristics of respondents and non-respondents. The availability of data on persistence in all patients eliminated a possible selection bias and also provides information that could be considered as more objective than (subjective) components of other methods of adherence measurement (i.e. self-reported questionnaires).

In summary, an association between psychiatric drug use and patients' beliefs about cardiovascular medication was found. The concomitant use of psychiatric drugs was associated with both increased beliefs about the importance of taking, but also greater concerns about, cardiovascular medication. While more attention might therefore be given in clinical practice to addressing such concerns, our study, however, found no association between concomitant psychiatric drug use and patients' persistence in taking cardiovascular medication.

\section{Acknowledgement}

Lucie Hromadkova is supported by Ministry of Health, Czech Republic - conceptual development of research organization (Nemocnice $\mathrm{Na}$ Homolce NNH, 00023884).

\section{Author contributions}

All listed authors sufficiently contributed to this manuscript and meet the criteria for authorship. All authors contributed to design of the study, data collection, analysis and interpretation, as well as to drafting article and its critical revision. All authors approved the article for publication. 


\section{References}

1 Nichols M, Townsend N, Scarborough P, Rayner M. Cardiovascular disease in Europe: epidemiological update. Eur Heart J 2013; 34: 3028-34.

2 Graham I, Atar D, Borch-Johnsen K et al. European guidelines on cardiovascular disease prevention in clinical practice: full text. Fourth Joint Task Force of the European Society of Cardiology and other societies on cardiovascular disease prevention in clinical practice (constituted by representatives of nine societies and by invited experts). Eur J Cardiovasc Prev Rehabil 2007; 14(Suppl. 2): S1-113.

3 DiMatteo MR, Giordani PJ, Lepper HS, Croghan TW. Patient adherence and medical treatment outcomes: a meta-analysis. Med Care 2002; 40: 794-811.

4 Horne R, Weinman J. Patients' beliefs about prescribed medicines and their role in adherence to treatment in chronic physical illness. J Psychosom Res 1999; 47: 555-67.

5 de Hert M, Correll CU, Bobes J et al. Physical illness in patients with severe mental disorders. I. Prevalence, impact of medications and disparities in health care. World Psychiatry 2011; 10: 52-77.

6 Casey DE. Metabolic issues and cardiovascular disease in patients with psychiatric disorders. Am J Med 2005; 118: 15S-22S.

7 Perlmutter JB, Frishman WH, Feinstein RE. Major depression as a risk factor for cardiovascular disease: therapeutic implications. Heart Dis 2000; 2: 75-82.

8 Jiang W, Krishnan RR, O'Connor CM. Depression and heart disease: evidence of a link, and its therapeutic implications. CNS Drugs 2002; 16: 111-27.

9 Rozanski A, Blumenthal JA, Kaplan J. Impact of psychological factors on the pathogenesis of cardiovascular disease and implications for therapy. Circulation 1999; 99: 2192-217.

10 Kawachi I, Sparrow D, Vokonas PS, Weiss ST. Symptoms of anxiety and risk of coronary heart disease. The Normative Aging Study. Circulation 1994; 90: 2225-9.

11 Hennekens CH, Hennekens AR, Hollar D, Casey DE. Schizophrenia and increased risks of cardiovascular disease. Am Heart J 2005; 150: 1115-21.

12 Nasrallah HA, Meyer JM, Goff DC et al. Low rates of treatment for hypertension, dyslipidemia and diabetes in schizophrenia: data from the CATIE schizophrenia trial sample at baseline. Schizophr Res 2006; 86: 15-22.

13 De Hert M, Falissard B, Mauri M, Shaw K, Wetterling T. Epidemiological study for the evaluation of metabolic disorders in patients with schizophrenia: the METEOR Study. Eur Neuropsychopharmacol 2008; 18(Suppl. 4): S444.
14 Laursen TM, Mortensen PB, MacCabe JH, Cohen D, Gasse C. Cardiovascular drug use and mortality in patients with schizophrenia or bipolar disorder: a Danish population-based study. Psychol Med 2014; 44: 1625-37.

15 Lahti M, Tiihonen J, Wildgust $\mathrm{H}$ et al. Cardiovascular morbidity, mortality and pharmacotherapy in patients with schizophrenia. Psychol Med 2012; 42 2275-85.

16 Desai MM, Rosenheck RA, Druss BG, Perlin JB. Mental disorders and quality of care among postacute myocardial infarction outpatients. J Nerv Ment Dis 2002; 190: 51-3.

17 Plomondon ME, Ho PM, Wang L et al. Severe mental illness and mortality of hospitalized ACS patients in the VHA. BMC Health Serv Res 2007; 7: 146.

18 Bautista LE, Vera-Cala LM, Colombo C, Smith P. Symptoms of depression and anxiety and adherence to antihypertensive medication. Am J Hypertens 2012; 25: 505-11.

19 Gehi A, Haas D, Pipkin S, Whooley MA. Depression and medication adherence in outpatients with coronary heart disease: findings from the Heart and Soul Study. Arch Intern Med 2005; 165: 2508-13.

20 Carney RM, Freedland KE, Eisen SA, Rich MW, Jaffe AS. Major depression and medication adherence in elderly patients with coronary artery disease. Health Psychol 1995; 14: 88-90.

21 Rieckmann N, Gerin W, Kronish IM et al. Course of depressive symptoms and medication adherence after acute coronary syndromes: an electronic medication monitoring study. J Am Coll Cardiol 2006; 48: 2218-22.

22 van Geffen EC, Philbert D, van Boheemen C, van Dijk L, Bos MB, Bouvy ML. Patients' satisfaction with information and experiences with counseling on cardiovascular medication received at the pharmacy. Patient Educ Couns 2011; 83: 303-9.

23 Horne R, Weinman J, Hankins M. The beliefs about medicines questionnaire: the development and evaluation of a new method for assessing the cognitive representation of medication. Psychol Health 1999; 14: 1-24.

24 Beaton DE, Bombardier C, Guillemin F, Ferray MB. Guidelines for the process of cross-cultural adaptation of self-report measures. Spine 2000; 25: 3186-91.

25 Sikka R, Xia F, Aubert RE. Estimating medication persistency using administrative claims data. Am J Manag Care 2005; 11: 449-57.

26 Cramer JA, Roy A, Burrell A et al. Medication compliance and persistence: terminology and definitions. Value Health 2008; 11: 44-7.
27 Hashord J, Mimran A, Simons WR. A populationbased European cohort study of persistence in newly diagnosed hypertensive patients. J Hum Hypertens 2002; 16: 569-75.

28 Glader E, Sjölander M, Eriksson M, Lundberg M. Persistent use of secondary preventive drugs declines rapidly during the first 2 years after stroke. Stroke 2010; 41: 397-401.

29 De las Cuevas C, Rivero-Santana A, PeresteloPerez L, Gonzalez-Lorenzo M, Perez-Ramos J, Sanz EJ. Adaptation and validation study of the Beliefs about Medicines Questionnaire in psychiatric outpatients in a community mental health setting. Hum Psychopharmacol 2011; 26: 140-6.

30 Cramer JA, Benedict A, Muszbek N, Keskinaslan A, Khan ZM. The significance of compliance and persistence in the treatment of diabetes, hypertension and dyslipidaemia: a review. Int J Clin Pract 2008; 62: 76-87.

31 Grosso G, Raciti T, Marventano S, Romeo I, Mistretta A. Adherence to antihypertensive and lipidlowering medications: a problem of public health, not yet resolved. Ann Ig 2011; 23: 173-84 [Abstract only].

32 Hashmi SK, Afridi MB, Abbas K et al. Factors associated with adherence to anti-hypertensive treatment in Pakistan. PLoS ONE 2007; 2: e280.

33 Krousel-Wood M, Thomas S, Muntner P, Morisky D. Medication adherence: a key factor in achieving blood pressure control and good clinical outcomes in hypertensive patients. Curr Opin Cardiol 2004; 19: 357-62.

34 Inkster ME, Donnan PT, MacDonald TM, Sullivan FM, Fahey T. Adherence to antihypertensive medication and association with patient and practice factors. J Hum Hypertens 2006; 20: 295-7.

35 Osterberg L, Blaschke T. Adherence to medication. N Engl J Med 2005; 353: 487-97.

36 Wang PS, Bohn RL, Knight E, Glynn RJ, Mogun $\mathrm{H}$, Avorn J. Noncompliance with antihypertensive medications. J Gen Intern Med 2002; 17: 504-11.

37 Kim MT, Han HR, Hill MN, Rose L, Roary M. Depression, substance use, adherence behaviors and blood pressure in urban hypertensive black men. Ann Behav Med 2003; 26: 24-31.

38 Gadkari AS, Pedan A, Gowda N, McHorney CA. Survey nonresponders to a medication-beliefs survey have worse adherence and persistence to chronic medications compared with survey responders. Med Care 2011; 49: 956-61.

Paper received June 2014, accepted August 2014 